J. Korean Math. Soc. 52 (2015), No. 4, pp. 765-780

http://dx.doi.org/10.4134/JKMS.2015.52.4.765

\title{
ON "VERY PALINDROMIC" SEQUENCES
}

\author{
BOJAN BAŠIĆ
}

\begin{abstract}
We consider the problem of characterizing the palindromic sequences $\left\langle c_{d-1}, c_{d-2}, \ldots, c_{0}\right\rangle, c_{d-1} \neq 0$, having the property that for any $K \in \mathbb{N}$ there exists a number that is a palindrome simultaneously in $K$ different bases, with $\left\langle c_{d-1}, c_{d-2}, \ldots, c_{0}\right\rangle$ being its digit sequence in one of those bases. Since each number is trivially a palindrome in all bases greater than itself, we impose the restriction that only palindromes with at least two digits are taken into account. We further consider a related problem, where we count only palindromes with a fixed number of digits (that is, $d$ ). The first problem turns out not to be very hard; we show that all the palindromic sequences have the required property, even with the additional point that we can actually restrict the counted palindromes to have at least $d$ digits. The second one is quite tougher; we show that all the palindromic sequences of length $d=3$ have the required property (and the same holds for $d=2$, based on some earlier results), while for larger values of $d$ we present some arguments showing that this tendency is quite likely to change.
\end{abstract}

\section{Introduction}

Whenever a property of a number depends on the notational base, it might be an interesting research direction to check whether a number can satisfy the considered property simultaneously in two or more bases, or even in each base, how many such numbers exist, how the possession of the property with respect to one base depends on the possession of the property with respect to another base etc. Let us mention a few interesting results of this kind.

Senge and Straus [17] proved that the number of integers such that the sum of its digits in each of the two bases $a$ and $b$ is smaller than a given bound is finite if and only if $\frac{\ln a}{\ln b} \notin \mathbb{Q}$; Stewart [18] gave an effective version of this result, and obtained a generalization. The independence (in a way) of the sumof-digits function in different bases was a subject of research of Bésineau [3],

Received September 9, 2014; Revised February 7, 2015.

2010 Mathematics Subject Classification. 11A63.

Key words and phrases. palindrome, number base, heuristic.

The research was supported by the Ministry of Education, Science and Technological Development of Serbia (project 174006) and by the Provincial Secretariat for Science and Technological Development, Autonomous Province of Vojvodina (project "Ordered structures and applications"). 
Kamae [10], Queffelec [15], Kim [11] etc. Mauduit, Pomerance and Sárközy [14] considered some questions about numbers that are Niven numbers with respect to multiple bases. In automata theory, Cobham [6] obtained a very interesting characterization of sets of integers recognizable independently of the notational base. Questions of this kind have been asked not only for integers, but also for real numbers. We mention, for example, the question when normality with respect to the base $a$ implies the normality with respect to the base $b$ (Schmidt [16]), the same question for disjunctiveness (El-Zanati and Transue [8]) and randomness (Calude and Jürgensen [4]) and so forth.

Hereby we are concerned with the property of being a palindrome in base $b$ (we call a number a palindrome in base $b$ if for its expansion in base $b$, say $\left\langle c_{d-1}, c_{d-2}, \ldots, c_{0}\right\rangle_{b}, c_{d-1} \neq 0$, the equality $c_{j}=c_{d-1-j}$ holds for every $j$ such that $0 \leqslant j \leqslant d-1)$. Various arithmetic properties of palindromes have been the subject of many works; for a few examples, see the introductory part of [2] (which is a paper that the present paper is a direct continuation of), and some more are $[1,12,13]$ and the very recent result [5], which states that for any base $b \geqslant 2$ and for any linear homogeneous recurrence sequence $\left(a_{n}\right)_{n \in \mathbb{N}}$ satisfying certain conditions there exists a positive constant $c>0$ such that $\mid\left\{n \leqslant x: a_{n}\right.$ is palindromic in base $\left.b\right\} \mid \in O\left(x^{1-c}\right)$.

Answering a question by Goins [9], the main result in [2] shows not only that for any given $K$ there is a number that is a palindrome simultaneously in $K$ different bases, but there is actually a number that is a $d$-digit palindrome simultaneously in $K$ different bases, where $d$ is given in advance. In fact, the same question has been asked by Di Scala and Sombra [7] a few years before Goins, but this was overlooked in the mentioned paper. The last section of [2] states a few questions that seem to be a natural continuation of research on this topic.

In particular, it is asked which palindromic sequences $\left\langle c_{d-1}, c_{d-2}, \ldots, c_{0}\right\rangle$, $c_{d-1} \neq 0$, have the property that for any $K \in \mathbb{N}$ there exists a number that is a $d$-digit palindrome simultaneously in $K$ different bases, with $\left\langle c_{d-1}, c_{d-2}, \ldots, c_{0}\right\rangle$ being its digit sequence in one of those bases. Is this true for the sequences $\langle 1,1,1\rangle$ and $\langle 1,0,1\rangle$ and, more generally, $\langle 1,1, \ldots, 1\rangle$ and $\langle 1,0,0, \ldots, 0,1\rangle$, is this perhaps true for all palindromic sequences, or could the sequences for which this is true be characterized? The only sequences for which this is known to be true are all the sequences

$$
\left\langle\left(\begin{array}{l}
d-1 \\
d-1
\end{array}\right),\left(\begin{array}{l}
d-1 \\
d-2
\end{array}\right),\left(\begin{array}{l}
d-1 \\
d-3
\end{array}\right), \ldots,\left(\begin{array}{c}
d-1 \\
1
\end{array}\right),\left(\begin{array}{c}
d-1 \\
0
\end{array}\right)\right\rangle
$$

where $d \geqslant 2$, as well as their multiples by a factor of the form $t^{d-1}$.

We could first ask a question that will turn out to be significantly easier: which palindromic sequences $\left\langle c_{d-1}, c_{d-2}, \ldots, c_{0}\right\rangle, c_{d-1} \neq 0$, have the property that for any $K \in \mathbb{N}$ there exists a number that is a palindrome (not necessarily with $d$ digits) simultaneously in $K$ different bases, with $\left\langle c_{d-1}, c_{d-2}, \ldots, c_{0}\right\rangle$ being its digit sequence in one of those bases? Of course, since each number is a 
(one-digit) palindrome in all the bases greater than itself, the question is trivial unless some restriction is imposed. Two restrictions that seem reasonable are either to take into account only palindromes having at least two digits, or to take into account only palindromes having at least $d$ digits. The first restriction is perhaps more natural, but in Section 2 we show that, even under the second restriction (which is a stronger one), actually all the palindromic sequence have the described property. In Sections 3 and 4 we consider the question from the previous paragraph; we show that all the palindromic sequences of length 3 have the property described there (and we recall that this is also true for the palindromic sequences of length 2), while for longer palindromic sequences we present some arguments showing that this tendency is quite likely to change. Finally, in Section 5 we present a few possible directions for further research.

\section{Variable number of digits}

The following theorem actually turns out to be easy to prove, but we nevertheless find it an interesting result. Note that (here and onward) $\mathbb{N}$ stands for the set of positive integers.

Theorem 2.1. Let $d \geqslant 2$ and a palindromic sequence $\left\langle c_{d-1}, c_{d-2}, \ldots, c_{0}\right\rangle$, $c_{d-1} \neq 0$, be given. Then for any $K \in \mathbb{N}$ there exist $n \in \mathbb{N}$ and a list of bases $\left\{b_{1}, b_{2}, \ldots, b_{K}\right\}$ such that, for each $i$ such that $1 \leqslant i \leqslant K, n$ is a palindrome with at least $d$ digits in base $b_{i}$, and that, for some $i_{0}$ such that $1 \leqslant i_{0} \leqslant K$, we have $\left\langle c_{d-1}, c_{d-2}, \ldots, c_{0}\right\rangle_{b_{i_{0}}}=n$.

Proof. Choose any $m \in \mathbb{N}$ that is greater than each $c_{i}$, and any $s \in \mathbb{N}$ that has (at least) $K$ divisors. Let $1=a_{1}, a_{2}, \ldots, a_{K}$ be the divisors of $s$. We claim that

$$
n=\sum_{j=0}^{d-1} c_{j} m^{s j}
$$

and the list $\left\{b_{i}: 1 \leqslant i \leqslant K\right\}$, where $b_{i}=m^{\frac{s}{a_{i}}}$, satisfy the given requirements. Indeed, for each $i$ such that $1 \leqslant i \leqslant K$ we have

$$
\langle c_{d-1}, \underbrace{0,0, \ldots, 0}_{a_{i}-1 \text { zeros }}, c_{d-2}, \underbrace{0,0, \ldots, 0}_{a_{i}-1 \text { zeros }}, c_{d-3}, 0,0, \ldots, 0,0, c_{1}, \underbrace{0,0, \ldots, 0}_{a_{i}-1 \text { zeros }}, c_{0}\rangle_{b_{i}}=n
$$

which can be seen by noting that

$$
\sum_{j=0}^{d-1} c_{j}\left(m^{\frac{s}{a_{i}}}\right)^{a_{i} j}=\sum_{j=0}^{d-1} c_{j} m^{s j}=n .
$$

Therefore, $n$ is indeed a palindrome in base $b_{i}$, and has $a_{i}(d-1)+1 \geqslant d-1+1=$ $d$ digits. Further, we have $\left\langle c_{d-1}, c_{d-2}, \ldots, c_{0}\right\rangle_{b_{1}}=n$, which completes the proof. 


\section{Three digits}

It is mentioned in Introduction that all the sequences of the form (1), as well as their multiples by a factor of the form $t^{d-1}$, have the property that we are interested in here. Note that for $d=2$ the sequence (1) becomes $\langle 1,1\rangle$; multiplying it by a factor of the form $t^{d-1}$ actually becomes multiplying by any factor $t$, which thus gives all the palindromic sequences of length 2 . We now show that also for $d=3$ all sequences fulfill the requirement.

Theorem 3.1. Let a palindromic sequence $\left\langle c_{0}, c_{1}, c_{0}\right\rangle, c_{0} \neq 0$, be given. Then for any $K \in \mathbb{N}$ there exist $n \in \mathbb{N}$ and a list of bases $\left\{b_{1}, b_{2}, \ldots, b_{K}\right\}$ such that, for each $i$ such that $1 \leqslant i \leqslant K, n$ is a 3 -digit palindrome in base $b_{i}$, and that, for some $i_{0}$ such that $1 \leqslant i_{0} \leqslant K$, we have $\left\langle c_{0}, c_{1}, c_{0}\right\rangle_{b_{i_{0}}}=n$.

Proof. We actually give two constructions that prove this theorem, based on completely different approaches. Since next to nothing is known in the case $d>3$, the author believes that offering two different approaches here increases chances for researches to build on some of these ideas and make a progress in the case $d>3$.

In both constructions we assume that a palindromic sequence $\left\langle c_{0}, c_{1}, c_{0}\right\rangle$, $c_{0} \neq 0$, and $K \in \mathbb{N}$ are given, and find $n \in \mathbb{N}$ and a list of bases $\left\{b_{1}, b_{2}, \ldots, b_{K}\right\}$ satisfying the statement.

\subsection{First construction}

Let $s$ be a number that is coprime to all the numbers $1,2, \ldots, K-1$ and to $c_{0}$ (we may, e.g., choose $s$ such that $s-1$ is a multiple of $c_{0}(K-1)$ !). We further require that $s$ is large enough, the meaning of which will be specified later.

Note that, for each $i$ such that $1 \leqslant i \leqslant K-1$, since $i$ is coprime to $s$, we also have that $i$ is coprime to $s-i c_{0}(K-2)$ !; for a similar reason, $(K-2)$ ! is coprime to $s-i c_{0}(K-2)$ !; finally, since $c_{0}$ is coprime to $s$, we also have that $c_{0}$ is coprime to $s-i c_{0}(K-2)$ !. Therefore, there exists modular inverse of $i c_{0}^{2}(K-2)$ ! modulo $s-i c_{0}(K-2)$ !. This shows that the right-hand sides of the following system of congruences are well defined:

$$
\begin{array}{ll}
m \equiv-\frac{c_{1}}{c_{0}^{2}(K-2) !} & \left(\bmod s-c_{0}(K-2) !\right), \\
m \equiv-\frac{c_{1}}{2 c_{0}^{2}(K-2) !} & \left(\bmod s-2 c_{0}(K-2) !\right), \\
m \equiv-\frac{c_{1}}{3 c_{0}^{2}(K-2) !} & \vdots \\
m \equiv-\frac{c_{1}}{(K-1) c_{0}^{2}(K-2) !} & \left(\bmod s-(K-1) c_{0}(K-2) !\right) .
\end{array}
$$


Since $s$ can be chosen as large as we want, we can achieve that all the moduli are positive. We now show that this system has a solution $m$. It is enough to prove that the moduli are pairwise coprime, since the Chinese remainder theorem then immediately gives the solution $m$. Suppose that $p \mid s-i_{1} c_{0}(K-2)$ ! and $p \mid s-i_{2} c_{0}(K-2)$ !, where $p$ is prime and $1 \leqslant i_{1}<i_{2} \leqslant K-1$. Then

$$
p \mid\left(s-i_{1} c_{0}(K-2) !\right)-\left(s-i_{2} c_{0}(K-2) !\right)=\left(i_{2}-i_{1}\right) c_{0}(K-2) ! .
$$

If $p \mid c_{0}$, then from $p \mid s-i_{1} c_{0}(K-2)$ ! we get $p \mid s$, which contradicts the fact that $s$ and $c_{0}$ are coprime. If $p \mid(K-2)$ !, then we similarly get $p \mid s$, but since $p \mid(K-2)$ ! implies $p \leqslant K-2$ and $s$ is coprime to all the numbers $1,2, \ldots, K-1$, this is again a contradiction. Finally, if $p \mid i_{2}-i_{1}$, then $p \leqslant i_{2}-i_{1} \leqslant K-2$ and thus $p \mid(K-2)$ !, which reduces this case to the previous one.

Therefore, let $m$ be a solution of the system (2). Note that we may choose a solution $m$ as large as we want, which will be needed. Finally, let

$$
n=c_{0}(m s)^{2}+c_{1} m s+c_{0} .
$$

We claim that $n$ is a 3 -digit palindrome in each base $b_{i}, 1 \leqslant i \leqslant K$, where

$$
b_{i}=m\left(s-(i-1) c_{0}(K-2) !\right) .
$$

The rightmost digit of $n$ in base $b$ equals $n \bmod b$. If $\left\lfloor\frac{n}{b^{2}}\right\rfloor<b$, then $n$ has at most 3 digits in base $b$, and its third digit from the right equals $\left\lfloor\frac{n}{b^{2}}\right\rfloor$. Therefore, $n$ is a 3-digit palindrome in base $b$ if and only if $n \bmod b=\left\lfloor\frac{n}{b^{2}}\right\rfloor$.

We now claim that for each $i, 2 \leqslant i \leqslant K$, we have

$$
s-(i-1) c_{0}(K-2) ! \mid c_{0} m s+c_{1} .
$$

Indeed:

$$
\begin{aligned}
c_{0} m s+c_{1} & \equiv c_{0} m\left((i-1) c_{0}(K-2) !\right)+c_{1} \\
& \equiv c_{0}\left(-\frac{c_{1}}{(i-1) c_{0}^{2}(K-2) !}\right)\left((i-1) c_{0}(K-2) !\right)+c_{1} \\
& =-\frac{c_{0}^{2} c_{1}(i-1)(K-2) !}{(i-1) c_{0}^{2}(K-2) !}+c_{1} \\
& =-c_{1}+c_{1} \\
& =0 \quad\left(\bmod s-(i-1) c_{0}(K-2) !\right) .
\end{aligned}
$$

Therefore, $b_{i} \mid m\left(c_{0} m s+c_{1}\right)=c_{0} m^{2} s+c_{1} m$, and thus

$$
b_{i} \mid s\left(c_{0} m^{2} s+c_{1} m\right)=c_{0}(m s)^{2}+c_{1} m s=n-c_{0} .
$$

This means that $n$ modulo $b_{i}$ equals $c_{0}$ for each $i$ such that $2 \leqslant i \leqslant K$. For $i=1$ we have $b_{1}=m s$, and thus again $n$ modulo $b_{i}$ equals $c_{0}$. (Recall that $m$ can be chosen large enough so that $b_{i}>c_{0}$.)

We now prove that $\left\lfloor\frac{n}{b_{i}^{2}}\right\rfloor=c_{0}$, that is, $c_{0} \leqslant \frac{n}{b_{i}^{2}}<c_{0}+1$. The first inequality is obvious. Let us now show the second one. It is enough to show that $\frac{n}{b_{i}^{2}}$ can 
arbitrarily close to $c_{0}$. As we observed earlier, for a fixed $s, m$ can be chosen as large as we want. Note that

$$
\begin{aligned}
\lim _{m \rightarrow \infty} \frac{n}{b_{i}^{2}} & =\lim _{m \rightarrow \infty} \frac{c_{0}(m s)^{2}+c_{1} m s+c_{0}}{\left(m\left(s-(i-1) c_{0}(K-2) !\right)\right)^{2}} \\
& =\lim _{m \rightarrow \infty} \frac{c_{0} s^{2}+\frac{c_{1} s}{m}+\frac{c_{0}}{m^{2}}}{\left(s-(i-1) c_{0}(K-2) !\right)^{2}} \\
& =\frac{c_{0} s^{2}}{\left(s-(i-1) c_{0}(K-2) !\right)^{2}} .
\end{aligned}
$$

Therefore, for any fixed value of $s$, the quotient $\frac{n}{b_{i}^{2}}$ can be arbitrarily close to

$$
\frac{c_{0} s^{2}}{\left(s-(i-1) c_{0}(K-2) !\right)^{2}} .
$$

Now, since $s$ can be chosen as large as we want, in order to prove the claim it is enough to note that

$$
\lim _{s \rightarrow \infty} \frac{c_{0} s^{2}}{\left(s-(i-1) c_{0}(K-2) !\right)^{2}}=\lim _{s \rightarrow \infty} \frac{c_{0}}{\left(1-\frac{(i-1) c_{0}(K-2) !}{s}\right)^{2}}=c_{0} .
$$

Finally, the observation that $b_{1}=m s$ and $\left\langle c_{0}, c_{1}, c_{0}\right\rangle_{b_{1}}=c_{0}(m s)^{2}+c_{1} m s+$ $c_{0}=n$ completes the proof of Theorem 3.1.

\subsection{Second construction}

Since some details in this construction depend on whether $c_{1}=0$ or not, we need to distinguish two cases.

The case $c_{1} \neq 0$. Let $p$ and $q$ be two positive integers such that: i) $\frac{\ln p}{\ln q} \notin \mathbb{Q}$; ii) $c_{0} \mid p q$; iii) $p q$ is even; iv) $p q \geqslant c_{1}$; v) $1<\frac{q}{p}<\sqrt{\frac{c_{0}+1}{c_{0}}}$. These requirements are given in order in which they are needed through the proof. We also note that such numbers indeed exists: one possibility is to take $p$ equal to a multiple of $c_{0}$ and take $q=p+1$, which satisfies the requirements i), ii) and iii), and by taking such $p$ large enough we can also fulfill iv) and v).

We first show that there exist nonnegative integers $g$ and $h$ such that

$$
1<\frac{p^{g}}{q^{h}}<\sqrt{\frac{c_{0}+1}{c_{0}}} .
$$

This means that we need $g$ and $h$ such that

$$
0<g \ln p-h \ln q<\ln \sqrt{\frac{c_{0}+1}{c_{0}}} .
$$

Since i) states that $\frac{\ln p}{\ln q} \notin \mathbb{Q}$, the sequence $\left(\left\{g \frac{\ln p}{\ln q}\right\}\right)_{g \in \mathbb{N}}$, where $\{\cdot\}$ denotes the fractional part, is equidistributed in the interval $[0,1]$. Therefore, we can choose 
$g \in \mathbb{N}$ such that

$$
0<\left\{g \frac{\ln p}{\ln q}\right\}<\frac{\ln \sqrt{\frac{c_{0}+1}{c_{0}}}}{\ln q}
$$

Now letting $h=\left\lfloor g \frac{\ln p}{\ln q}\right\rfloor$ leads to

$$
g \ln p-h \ln q=\ln q\left(g \frac{\ln p}{\ln q}-h\right)=\ln q\left(g \frac{\ln p}{\ln q}-\left\lfloor g \frac{\ln p}{\ln q}\right\rfloor\right)=\ln q\left\{g \frac{\ln p}{\ln q}\right\},
$$

and (3) follows by (4).

We now choose large enough positive integer $M$ such that $(p q+1)^{M} \geqslant$ $4\left(\left\lceil\frac{g+1}{2}\right\rceil+K\right)+1,(p q+1)^{M} \geqslant h+1$ and $M \geqslant\left\lceil\frac{g-1}{2}\right\rceil+K$. Finally, let

$$
a=\frac{c_{1}(p q)^{(p q+1)^{M}}}{c_{0}}
$$

(note that $a$ is an integer because of ii)), and let

$$
n=c_{0} a^{2}+c_{1} a+c_{0} .
$$

We shall now find $K$ bases $b_{1}, b_{2}, \ldots, b_{K}$ such that $n$ is a 3 -digit palindrome in all those bases. We take $b_{1}=a$, which gives $n=\left\langle c_{0}, c_{1}, c_{0}\right\rangle_{b_{1}}$. Let us now construct $K-1$ more bases.

In fact, we shall find such bases that $n$ begins and ends with $c_{0}$ when written in each of these bases. Note that the requirement $n=\left\langle c_{0}, f, c_{0}\right\rangle_{b}=c_{0} b^{2}+f b+c_{0}$ can be transformed to $b\left(c_{0} b+f\right)=n-c_{0}=a\left(c_{0} a+c_{1}\right)$. Therefore, any divisor $b$ of $a\left(c_{0} a+c_{1}\right)$, larger than $c_{0}$ and $c_{1}$, such that $\frac{a\left(c_{0} a+c_{1}\right)}{b}$ equals $c_{0} b+f$ with $0 \leqslant f<b$, that is, that

$$
c_{0} b \leqslant \frac{a\left(c_{0} a+c_{1}\right)}{b}<\left(c_{0}+1\right) b
$$

represents a base that satisfies the requirement. The last double inequality reduces to

$$
\sqrt{\frac{a\left(c_{0} a+c_{1}\right)}{c_{0}+1}}<b \leqslant \sqrt{\frac{a\left(c_{0} a+c_{1}\right)}{c_{0}}} .
$$

Let us show that there indeed exist $K-1$ divisors of $a\left(c_{0} a+c_{1}\right)$ that satisfy this inequality.

Recall the following well-known fact (easily proved by induction): if $x$ is an odd integer and $x \mid y+1$, then $x^{j} \mid y^{x^{j-1}}+1$ for each $j$. Since, by iii), $p q+1$ is odd, for $x=p q+1, y=p q$ and $j=M+1$ we get

$$
(p q+1)^{M+1} \mid(p q)^{(p q+1)^{M}}+1=\frac{c_{0} a}{c_{1}}+1 .
$$


We shall now show that for each $i$ such that $\left\lceil\frac{g+5}{2}\right\rceil \leqslant i \leqslant\left\lceil\frac{g+1}{2}\right\rceil+K$ there exist $u_{i}, v_{i}$ such that $0 \leqslant u_{i}, v_{i} \leqslant(p q+1)^{M}-1$ and

$$
\frac{\sqrt{\frac{a\left(c_{0} a+c_{1}\right)}{c_{0}+1}}}{(p q+1)^{i}}<p^{u_{i}} q^{v_{i}} \leqslant \frac{\sqrt{\frac{a\left(c_{0} a+c_{1}\right)}{c_{0}}}}{(p q+1)^{i}} .
$$

Let $i$ be fixed, $\left\lceil\frac{g+5}{2}\right\rceil \leqslant i \leqslant\left\lceil\frac{g+1}{2}\right\rceil+K$, and let

$$
z=\frac{\sqrt{a\left(c_{0} a+c_{1}\right)}}{(p q+1)^{i}} .
$$

Then (7) reduces to

$$
\frac{z}{\sqrt{c_{0}+1}}<p^{u_{i}} q^{v_{i}} \leqslant \frac{z}{\sqrt{c_{0}}}
$$

Let $w$ be the largest integer such that $(p q)^{w} \leqslant \frac{z}{\sqrt{c_{0}}}$, that is, $w=\left\lfloor\log _{p q} \frac{z}{\sqrt{c_{0}}}\right\rfloor$. Since

$$
\begin{aligned}
\frac{z}{\sqrt{c_{0}}} & =\frac{\sqrt{a\left(c_{0} a+c_{1}\right)}}{(p q+1)^{i} \sqrt{c_{0}}}>\frac{a}{(p q+1)^{i}}>\frac{a}{(2 p q)^{i}}>\frac{a}{(p q)^{2 i}} \\
& =\frac{c_{1}(p q)^{(p q+1)^{M}-2 i}}{c_{0}} \geqslant(p q)^{(p q+1)^{M}-2 i-1}
\end{aligned}
$$

(the relation ii) indeed implies that $c_{0} \leqslant p q$ ) and

$$
\begin{aligned}
\frac{z}{\sqrt{c_{0}}} & =\frac{\sqrt{a\left(c_{0} a+c_{1}\right)}}{(p q+1)^{i} \sqrt{c_{0}}}<\frac{\sqrt{a\left(c_{0} a+p q c_{0} a\right)}}{(p q+1)^{i} \sqrt{c_{0}}}=\frac{a \sqrt{c_{0}}}{(p q+1)^{i-\frac{1}{2}} \sqrt{c_{0}}}<\frac{a}{(p q)^{i-\frac{1}{2}}} \\
& =\frac{c_{1}(p q)^{(p q+1)^{M}-i+\frac{1}{2}}}{c_{0}} \stackrel{\text { iv })}{\leqslant}(p q)^{(p q+1)^{M}-i+\frac{1}{2}+1}<(p q)^{(p q+1)^{M}-i+2},
\end{aligned}
$$

we get

$$
(p q+1)^{M}-2 i-1 \leqslant w \leqslant(p q+1)^{M}-i+1 .
$$

Let $l=1$ if $p^{w} q^{w+1} \leqslant \frac{z}{\sqrt{c_{0}}}$, and $l=0$ otherwise. In any case we have $p^{w} q^{w+l} \leqslant$ $\frac{z}{\sqrt{c_{0}}}$ and $p^{w} q^{w+l+1}>\frac{z}{\sqrt{c_{0}}}$ (if $l=0$ this follows by the choice of $l$, while if $l=1$ this follows because $p^{w} q^{w+2}>(p q)^{w+1}>\frac{z}{\sqrt{c_{0}}}$, by the choice of $\left.w\right)$. If $p^{w} q^{w+l}>\frac{z}{\sqrt{c_{0}+1}}$, we may choose $u_{i}=w, v_{i}=w+l$, and we have (8), as needed. Thus, let us assume that $p^{w} q^{w+l} \leqslant \frac{z}{\sqrt{c_{0}+1}}$. We shall now describe a procedure that starts from the number $p^{w} q^{w+l}$ and repeatedly multiplies it by a factor less than $\sqrt{\frac{c_{0}+1}{c_{0}}}$, all the time keeping the exponents of $p$ and $q$ bounded between 0 and $(p q+1)^{M}-1$ inclusively; after a finite number of steps we shall obtain a number greater than $\frac{z}{\sqrt{c_{0}}}$. Since in each step the current number is multiplied by a factor less than $\sqrt{\frac{c_{0}+1}{c_{0}}}$, and since the leftmost and 
the rightmost side of $(8)$ differ by a factor of $\sqrt{\frac{c_{0}+1}{c_{0}}}$, this will mean that during the procedure a number that satisfies (8) is encountered.

We first repeatedly multiply by the factor $\frac{q}{p}$ (note the bounds v)) until the exponent of $q$ becomes equal to $(p q+1)^{M}-1$. Since the sum of the exponents of $p$ and $q$ remains constant (equal to $2 w+l$ ) during this multiplying, the number obtained at the end is

$$
p^{2 w+l-(p q+1)^{M}+1} q^{(p q+1)^{M}-1} .
$$

Let us check that the exponent of $p$ is nonnegative. Since

$$
w \geqslant(p q+1)^{M}-2 i-1 \geqslant(p q+1)^{M}-2\left(\left\lceil\frac{g+1}{2}\right\rceil+K\right)-1,
$$

we have

$$
2 w+l-(p q+1)^{M}+1 \geqslant(p q+1)^{M}-4\left(\left\lceil\frac{g+1}{2}\right\rceil+K\right)-1 \geqslant 0,
$$

where the latter inequality follows by the choice of $M$. Let us now multiply the number (9) by $\frac{p^{g}}{q^{h}}$ only once, and thus get the number

$$
p^{2 w+l-(p q+1)^{M}+1+g} q^{(p q+1)^{M}-1-h} .
$$

We check that the exponent of $q$ is nonnegative, and that the exponent of $p$ is less than or equal to $(p q+1)^{M}-1$. The first claim follows immediately by the choice of $M$. Regarding the second claim, since

$$
\begin{aligned}
w \leqslant(p q+1)^{M}-i+1 & \leqslant(p q+1)^{M}-\left\lceil\frac{g+5}{2}\right\rceil+1 \\
& =(p q+1)^{M}-\left\lceil\frac{g+3}{2}\right\rceil,
\end{aligned}
$$

we have

$$
\begin{aligned}
2 w+l-(p q+1)^{M}+1+g & \leqslant(p q+1)^{M}-2\left\lceil\frac{g+3}{2}\right\rceil+1+1+g \\
& \leqslant(p q+1)^{M}-2 \cdot \frac{g+3}{2}+2+g \\
& \leqslant(p q+1)^{M}-1,
\end{aligned}
$$

as needed. Finally, we multiply the number (10) by $\frac{q}{p}$ repeatedly $h$ times. We thus get the number

$$
p^{2 w+l-(p q+1)^{M}+1+g-h} q^{(p q+1)^{M}-1} .
$$

Since, by the upper bound for $w$, we have the inequality

$$
\begin{aligned}
(p q+1)^{M}-1 & \geqslant w+i-2 \geqslant w+\left\lceil\frac{g+5}{2}\right\rceil-2=w+\left\lceil\frac{g+1}{2}\right\rceil \\
& \geqslant w+2 \geqslant w+l+1,
\end{aligned}
$$


we get

$$
\begin{aligned}
& p^{2 w+l-(p q+1)^{M}+1+g-h} q^{(p q+1)^{M}-1} \\
> & p^{2 w+l-(p q+1)^{M}+1+g-h+(p q+1)^{M}-1-w-l-1} q^{w+l+1} \\
= & p^{w+g-h-1} q^{w+l+1} \\
\geqslant & p^{w+1-1} q^{w+l+1} \\
= & p^{w} q^{w+l+1}>\frac{z}{\sqrt{c_{0}}} .
\end{aligned}
$$

The argument that shows the existence of $u_{i}, v_{i}$ from $(7)$ is thus finished.

Let us now complete the proof. By (7) we get

$$
\sqrt{\frac{a\left(c_{0} a+c_{1}\right)}{c_{0}+1}}<p^{u_{i}} q^{v_{i}}(p q+1)^{i} \leqslant \sqrt{\frac{a\left(c_{0} a+c_{1}\right)}{c_{0}}}
$$

for each $i$ such that $\left\lceil\frac{g+5}{2}\right\rceil \leqslant i \leqslant\left\lceil\frac{g+1}{2}\right\rceil+K$. Since $0 \leqslant u_{i}, v_{i} \leqslant(p q+1)^{M}-1$, by the definition of $a$ and the fact that $c_{0} \mid p q$ we get $p^{u_{i}} q^{v_{i}} \mid a$. Further, since $i \leqslant\left\lceil\frac{g+1}{2}\right\rceil+K \leqslant M+1$, by (6) we get $(p q+1)^{i} \mid c_{0} a+c_{1}$. Altogether,

$$
p^{u_{i}} q^{v_{i}}(p q+1)^{i} \mid a\left(c_{0} a+c_{1}\right) .
$$

Since there is a total of $K-1$ values of $i$ in the given range, we have thus found $K-1$ divisors of $a\left(c_{0} a+c_{1}\right)$ for which (5) holds, which was to be done. This completes the proof in the case $c_{1} \neq 0$.

The case $\boldsymbol{c}_{\mathbf{1}}=\mathbf{0}$. The underlying idea is actually very similar in this case, but much less technically demanding. Let $p$ and $q$ be two positive integers such that

$$
1<\frac{q}{p}<\left(\frac{c_{0}+1}{c_{0}}\right)^{\frac{1}{2 K-2}}
$$

let

$$
a=(p q)^{K-1}
$$

and let

$$
n=c_{0} a^{2}+c_{0}
$$

We claim that $n$ is a 3 -digit palindrome in each base $b_{i}, 1 \leqslant i \leqslant K$, where

$$
b_{i}=p^{K+i-2} q^{K-i} .
$$

As in the previous case, it is enough to show that each $b_{i}$ is a divisor of $a\left(c_{0} a+c_{1}\right)=a^{2} c_{0}$ such that

$$
a \sqrt{\frac{c_{0}}{c_{0}+1}}<b_{i} \leqslant a
$$


(this is the inequality (5) for $c_{1}=0$ ). And indeed, since $K+i-2 \leqslant 2 K-2$ and $K-i \geqslant 0$, we get that $b_{i}$ is a divisor of $a^{2}$, and hence also of $a^{2} c_{0}$. Further,

$$
b_{i}=p^{K+i-2} q^{K-i}=\frac{(p q)^{K-1} p^{i-1}}{q^{i-1}}=\frac{(p q)^{K-1}}{\left(\frac{q}{p}\right)^{i-1}}=\frac{a}{\left(\frac{q}{p}\right)^{i-1}},
$$

and thus $b_{i} \leqslant a$ and

$$
b_{i} \geqslant \frac{a}{\left(\frac{q}{p}\right)^{K-1}}>\frac{a}{\left(\left(\frac{c_{0}+1}{c_{0}}\right)^{\frac{1}{2 K-2}}\right)^{K-1}}=\frac{a}{\sqrt{\frac{c_{0}+1}{c_{0}}}}=a \sqrt{\frac{c_{0}}{c_{0}+1}},
$$

which proves (11). Finally, the observation that $b_{1}=a$ and $\left\langle c_{0}, 0, c_{0}\right\rangle_{b_{1}}=$ $c_{0} a^{2}+c_{0}=n$ completes the proof of Theorem 3.1.

\subsection{Examples and comparison of the two constructions}

In order to make these two constructions easier to grasp, we shall now present their outputs on two explicit examples. We then compare some of their aspects.

Let us first consider the sequence $\langle 1,5,1\rangle$ and $K=4$. For the first construction we first need $s$ that is coprime to 2 and 3 , and large enough so that $s-3 \cdot 1 \cdot 2 !>0$ and $\left\lfloor\frac{1 \cdot s^{2}}{(s-3 \cdot 1 \cdot 2 !)^{2}}\right\rfloor=c_{0}=1$. The smallest such $s$ is $s=23$. The system $(2)$ becomes $m \equiv-55(\bmod 21), m \equiv-25(\bmod 19)$ and $m \equiv-15$ $(\bmod 17)$, which has the solution $m \equiv 2654(\bmod 6783)$. We need $m$ large enough so that $\left\lfloor\frac{1 \cdot(23 m)^{2}+115 m+1}{(17 m)^{2}}\right\rfloor=1 ; m=2654$ suffices. We finally set

$$
n=c_{0}(m s)^{2}+c_{1} m s+c_{0}=3726430975,
$$

for which indeed

$$
n=\langle 1,5,1\rangle_{61042}=\langle 1,11127,1\rangle_{55734}=\langle 1,23473,1\rangle_{50426}=\langle 1,37475,1\rangle_{45118},
$$

where $61042=2654 \cdot 23,55734=2654 \cdot 21,50426=2654 \cdot 19$ and $45118=$ $2654 \cdot 17$.

Let us now see what the second construction gives for the same input. Choosing $p=3$ and $q=4$ fulfills all the requirements. We then may take $g=4$ and $h=3$, and after that $M=6$. Finally, let

$$
a=5 \cdot 12^{13^{6}}=2^{9653618} \cdot 3^{4826809} \cdot 5=\underbrace{28185 \cdots 21760}_{5209003 \text { digits }}
$$

and

$$
n=a^{2}+5 a+1=\underbrace{79441 \cdots 06401}_{10418005 \text { digits }} .
$$

For each $i$ such that $\left\lceil\frac{4+5}{2}\right\rceil \leqslant i \leqslant\left\lceil\frac{4+1}{2}\right\rceil+4$, that is, $5 \leqslant i \leqslant 7$, we use the described procedure in order to find $u_{i}, v_{i}$ for which $(7)$ holds. For $i=5$ we first find $w=4826804, l=0$, and after a few steps we ultimately get $3^{4826801} \cdot 4^{4826807} \cdot 13^{5} \mid a(a+5)$; for $i=6$ we get $w=4826803, l=0$ and 
ultimately $3^{4826800} \cdot 4^{4826806} \cdot 13^{6} \mid a(a+5)$, and for $i=7$ we get $w=4826802$, $l=0$ and ultimately $3^{4826799} \cdot 4^{4826805} \cdot 13^{7} \mid a(a+5)$. We conclude:

$$
\begin{aligned}
n & =\langle 1,5,1\rangle_{2^{9653618.3^{4} 826809.5}} \\
& =\langle 1, \underbrace{19906 \cdots 06864}_{5209003 \text { digits }}, 1\rangle_{2^{9653614.3^{4}} 826801.13^{5}} \\
& =\langle 1, \underbrace{15179 \cdots 59936}_{5209003 \text { digits }}, 1\rangle_{2^{9} 653612.3^{4} 826800.13^{6}} \\
& =\langle 1, \underbrace{10550 \cdots 83264}_{5209003 \text { digits }}, 1\rangle_{2^{9} 653610.3^{4826799.13^{7}} .}
\end{aligned}
$$

Since the second construction depends on whether $c_{1} \neq 0$ or not, we also check what happens for some example where $c_{1}=0$. Consider the sequence $\langle 2,0,2\rangle$ and $K=4$. We then may take $p=15$ and $q=16$, and after that $a=(15 \cdot 16)^{3}=13824000$. We finally set

$$
n=c_{0} a^{2}+c_{0}=382205952000002,
$$

for which indeed

$$
\begin{aligned}
n & =\langle 2,0,2\rangle_{13824000}=\langle 2,3571200,2\rangle_{12960000} \\
& =\langle 2,7157280,2\rangle_{12150000}=\langle 2,10773182,2\rangle_{11390625},
\end{aligned}
$$

where $13824000=15^{3} \cdot 16^{3}, 12960000=15^{4} \cdot 16^{2}, 12150000=15^{5} \cdot 16$ and $11390625=15^{6}$. For the record, the first construction for this input gives

$$
\begin{aligned}
n=375223562302052 & =\langle 2,0,2\rangle_{13697145}=\langle 2,3374800,2\rangle_{12879405} \\
& =\langle 2,6985440,2\rangle_{12061665}=\langle 2,10883376,2\rangle_{11243925} .
\end{aligned}
$$

Let us now try to make some conclusions.

Based on the first example, one gets the impression that the second construction is "worse" than the first one, in the sense that it produces a much larger value of $n$ than the first construction. However, this is not always the case, as the second example illustrates. And in fact, if $c_{1}=0$, then for larger values of $K$ the second construction indeed turns out to produce quite smaller values of $n$ than the first one: for example, for the sequence $\langle 2,0,2\rangle$ and $K=20$, the second construction produces a 151-digit number $n$, while the first one produces a 724-digit number $n$; for $K=100$ we get a 1066-digit $n$ from the second construction versus a 31394 -digit $n$ from the first construction.

Furthermore, the idea the second construction relies on leaves much space for improvement. Indeed, the core of the construction is finding a number $a$ such that the number $a\left(c_{0} a+c_{1}\right)$ has at least $K-1$ divisors $b$ for which the bounds (5) hold. It seems very plausible that, using some other techniques, this can be shown to hold for some number $a$ that is much smaller than the one we proposed here.

Finally, the main motivation for providing two different constructions for the case $d=3$ here is, as we have already mentioned, the belief that this increases 
chances of making some progress on the case $d>3$ by some modification of one of the presented constructions. There is no reason to believe that the construction that has the biggest chances of being useful for the case $d>3$ is the one that produces the smallest values of $n$ in the case $d=3$. In fact, as we shall see in the following section, there are some arguments that suggest that for $d>3$ the numbers we are looking for become much rarer; thus, it is not at all impossible that a construction that produces large values in the case $d=3$ can be adapted to be of some use also for $d>3$, while the one that produces small values in the case $d=3$ actually only picks some exceptions whose existence essentially relies on the assumption that $d=3$.

\section{Some heuristic arguments for the cases of more digits}

We present some heuristic reasons that seem to indicate that the situation might change for $d>3$, that is, that not every palindromic sequence $\left\langle c_{d-1}, c_{d-2}, \ldots, c_{0}\right\rangle, c_{d-1} \neq 0$, has the property that for any $K \in \mathbb{N}$ there exists a number that is a $d$-digit palindrome simultaneously in $K$ different bases, with $\left\langle c_{d-1}, c_{d-2}, \ldots, c_{0}\right\rangle$ being its digit sequence in one of those bases. For the sake of simplicity, we present our argument for the sequence $\langle 1,0,0, \ldots, 0,1\rangle$.

Note that the probability that a randomly chosen $d$-digit integer written in base $b$ is a palindrome equals $\frac{1}{b^{\lfloor d / 2\rfloor}}$. Let $n=a^{d-1}+1=\langle 1,0,0, \ldots, 0,1\rangle_{a}$. Then $n$ is a $d$-digit number in base $b$ if and only if $\lfloor\sqrt[d]{n}\rfloor+1 \leqslant b \leqslant a$. We want to estimate for how many of the bases $b$ from this range, apart from $b=a$, the number $n$ is a palindrome in base $b$. Assuming that the probability that $n$ is a palindrome in base $b$ equals $\frac{1}{b\lfloor d / 2]}$ for each $b$ from the given range independently, we get that the expected number of $d$-digit palindromic expansions of $n$ in base $b$, apart from $n=\langle 1,0,0, \ldots, 0,1\rangle_{a}$, equals

$$
\sum_{b=\lfloor\sqrt[d]{n}\rfloor+1}^{a-1} \frac{1}{b^{\left\lfloor\frac{d}{2}\right\rfloor}}=\sum_{b=\left\lfloor\sqrt[d]{a^{d-1}+1}\right\rfloor+1}^{a-1} \frac{1}{b^{\left\lfloor\frac{d}{2}\right\rfloor}} \leqslant \sum_{b=\left\lceil a^{\frac{d-1}{d}}\right\rceil}^{a-1} \frac{1}{b^{\left\lfloor\frac{d}{2}\right\rfloor}} .
$$

Therefore, the total number of $d$-digit palindromic expansions of numbers that are written as $\langle 1,0,0, \ldots, 0,1\rangle_{a}$ in some base $a$, where $a$ is bounded above by some bound $A$, not counting the expansions $\langle 1,0,0, \ldots, 0,1\rangle_{a}$ themselves, has the expected value bounded above by

$$
\sum_{a=2}^{A} \sum_{b=\left\lceil a^{\frac{d-1}{d}}\right\rceil}^{a-1} \frac{1}{b^{\left\lfloor\frac{d}{2}\right\rfloor}}
$$

We shall now transform the above expression in a more convenient form. Note that, if $a$ and $b$ are integers, then $b \geqslant\left\lceil a^{\frac{d-1}{d}}\right\rceil$ is equivalent to $b \geqslant a^{\frac{d-1}{d}}$, which 
B. BAŠIĆ

is equivalent to $a \leqslant b^{\frac{d}{d-1}}$, which is equivalent to $a \leqslant\left\lfloor b^{\frac{d}{d-1}}\right\rfloor$. We have

$$
\begin{aligned}
\sum_{a=2}^{A} \sum_{b=\left\lceil a^{\frac{d-1}{d}}\right\rceil}^{a-1} \frac{1}{b^{\left\lfloor\frac{d}{2}\right\rfloor}} & =\sum_{b=\left\lceil 2^{\frac{d-1}{d}}\right\rceil}^{A-1} \frac{1}{b^{\left\lfloor\frac{d}{2}\right\rfloor}} \mid\left\{a: 2 \leqslant a \leqslant A \text { and }\left\lceil a^{\frac{d-1}{d}}\right\rceil \leqslant b \leqslant a-1\right\} \mid \\
& =\sum_{b=2}^{A-1} \frac{1}{b^{\left\lfloor\frac{d}{2}\right\rfloor}} \mid\left\{a: 2 \leqslant a \leqslant A \text { and } b+1 \leqslant a \leqslant\left\lfloor b^{\frac{d}{d-1}}\right\rfloor\right\} \mid \\
& =\sum_{b=2}^{A-1} \frac{1}{b^{\left\lfloor\frac{d}{2}\right\rfloor}}\left|\left\{a: b+1 \leqslant a \leqslant \min \left\{A,\left\lfloor b^{\frac{d}{d-1}}\right\rfloor\right\}\right\}\right| \\
& =\sum_{b=2}^{A-1} \frac{\min \left\{A,\left\lfloor b^{\frac{d}{d-1}}\right\rfloor\right\}-(b+1)+1}{b^{\left\lfloor\frac{d}{2}\right\rfloor}} \\
& =\sum_{b=2}^{A-1} \frac{\min \left\{A,\left\lfloor b^{\frac{d}{d-1}}\right\rfloor\right\}-b}{b^{\left\lfloor\frac{d}{2}\right\rfloor}} \\
& \leqslant \sum_{b=2}^{A-1} \frac{b^{\frac{d}{d-1}}-b}{b^{\left\lfloor\frac{d}{2}\right\rfloor}}=\sum_{b=2}^{A-1} \frac{1}{b^{\left\lfloor\frac{d}{2}\right\rfloor-\frac{d}{d-1}}}-\sum_{b=2}^{A-1} \frac{1}{b^{\left\lfloor\frac{d}{2}\right\rfloor-1}} .
\end{aligned}
$$

Note that for $d \geqslant 6$ the inequalities $\left\lfloor\frac{d}{2}\right\rfloor-\frac{d}{d-1}>1$ and $\left\lfloor\frac{d}{2}\right\rfloor-1>1$ hold. Therefore, for $A \rightarrow \infty$ the above value actually converges to

$$
\zeta\left(\left\lfloor\frac{d}{2}\right\rfloor-\frac{d}{d-1}\right)-\zeta\left(\left\lfloor\frac{d}{2}\right\rfloor-1\right) .
$$

This provides some heuristic evidence that for $d \geqslant 6$ there are in fact only finitely many numbers that are written as $\langle 1,0,0, \ldots, 0,1\rangle$ (a total of $d$ digits) in some base, and that are $d$-digit palindromes also in some other base. For $d \in\{4,5\}$ the same heuristic suggests that such numbers, though there might be infinitely many of them, are very rare.

\section{Future directions}

By "very palindromic" sequences, let us refer to the palindromic sequences $\left\langle c_{d-1}, c_{d-2}, \ldots, c_{0}\right\rangle, c_{d-1} \neq 0$, such that for any $K \in \mathbb{N}$ there exists a number that is a $d$-digit palindrome simultaneously in $K$ different bases, with $\left\langle c_{d-1}, c_{d-2}, \ldots, c_{0}\right\rangle$ being its digit sequence in one of those bases. Of course, the main question in this topic is the following one.

Open problem 1. Characterize all "very palindromic" sequences for $d>3$.

If this problem turns out to be too hard, some simpler starting points might be the following problems.

Open problem 2. Are sequences $\langle 1,1, \ldots, 1\rangle$ and $\langle 1,0,0, \ldots, 0,1\rangle$ "very palindromic" (for any $d$, or for each $d$ )? 
Open problem 3. Provide at least a single example of a "very palindromic" sequence, other than the "known sequences" (for $d>3$ ), or prove that there are not any. (By "known sequences" we mean the sequences of the form (1), as well as their multiples by a factor of the form $t^{d-1}$.)

Open problem 4. Provide at least a single example of a palindromic sequence that is not "very palindromic" (for $d>3$ ), or prove that there are not any.

If palindromic sequences that are not "very palindromic" were found, a further research direction could be to determine, for a given such sequence, what the largest $K \in \mathbb{N}$ is such that there exists a number that is a $d$-digit palindrome simultaneously in $K$ different bases, with the given sequence being its digit sequence in one of those bases. In particular, could such $K$ be equal to 1 for some sequence? If the answer is affirmative, such sequences can be intuitively thought of as "very nonpalindromic", that is, they would be at exactly the opposite end of the scale in comparison to "very palindromic" sequences.

\section{References}

[1] W. D. Banks and I. E. Shparlinski, Average value of the Euler function on binary palindromes, Bull. Pol. Acad. Sci. Math. 54 (2006), no. 2, 95-101.

[2] B. Bašić, On d-digit palindromes in different bases: The number of bases is unbounded, Int. J. Number Theory 8 (2012), no. 6, 1387-1390.

[3] J. Bésineau, Indépendance statistique d'ensembles liés á la fonction "somme des chiffre", Acta Arith. 20 (1972), 401-416.

[4] C. Calude and H. Jürgensen, Randomness as an invariant for number representations, in: H. Maurer, J. Karhumäki and G. Rozenberg (Eds.), Results and trends in theoretical computer science, pp. 44-66, Springer-Verlag, Berlin, 1994.

[5] J. Cilleruelo, R. Tesoro, and F. Luca, Palindromes in linear recurrence sequences, Monatsh. Math. 171 (2013), no. 3-4, 433-442.

[6] A. Cobham, On the base-dependence of sets of numbers recognizable by finite automata, Math. Systems Theory 3 (1969), 186-192.

[7] A. J. Di Scala and M. Sombra, Intrinsic palindromes, Fibonacci Quart. 42 (2004), no. $1,76-81$.

[8] S. I. El-Zanati and W. R. R. Transue, On dynamics of certain Cantor sets, J. Number Theory 36 (1990), no. 2, 246-253.

[9] E. H. Goins, Palindromes in different bases: A conjecture of J. Ernest Wilkins, Integers 9 (2009), 725-734.

[10] T. Kamae, Sum of digits to different bases and mutual singularity of their spectral measures, Osaka J. Math. 15 (1978), no. 3, 569-574.

[11] D.-H. Kim, On the joint distribution of $q$-additive functions in residue classes, J. Number Theory 74 (1999), no. 2, 307-336.

[12] F. Luca, Palindromes in Lucas sequences, Monatsh. Math. 138 (2003), no. 3, 209-223.

[13] F. Luca and A. Togbé, On binary palindromes of the form $10^{n} \pm 1$, C. R. Math. Acad. Sci. Paris 346 (2008), no. 9-10, 487-489.

[14] C. Mauduit, C. Pomerance, and A. Sárközy, On the distribution in residue classes of integers with a fixed sum of digits, Ramanujan J. 9 (2005), no. 1-2, 45-62.

[15] M. Queffelec, Sur la singularité des produits de Riesz et des mesures spectrales associées á la somme des chiffres, Israel J. Math. 34 (1979), no. 4, 337-342.

[16] W. M. Schmidt, On normal numbers, Pacific J. Math. 10 (1960), 661-672. 
[17] H. G. Senge and E. G. Straus, PV-numbers and sets of multiplicity, in: Proceedings of the Washington State University Conference on Number Theory, pp. 55-67, Dept. Math., Washington State Univ., Pullman, Washington, 1971.

[18] C. L. Stewart, On the representation of an integer in two different bases, J. Reine Angew. Math. 319 (1980), 63-72.

Department of Mathematics AND Informatics

UNIVERSITY OF NOVI SAD

Trg Dositeja Obradovića 4

21000 Novi Sad, Serbia

E-mail address: bojan.basic@dmi.uns.ac.rs 